The INL is a

U.S. Department of Energy

National Laboratory

operated by

Battelle Energy Alliance

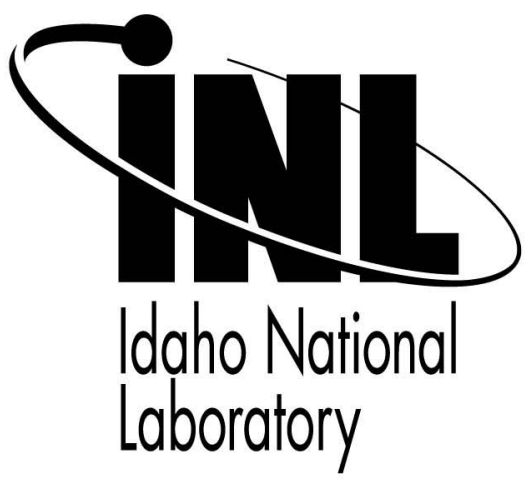

INL/CON-06-11118

PREPRINT

\title{
Quantitative
}

\section{Determination of Lateral Mode Dispersion in Film Bulk Acoustic Resonators Through Laser Acoustic Imaging}

\section{IEEE International Ultrasonics Symposium}

\author{
Ken L. Telschow \\ John D. Larson III
}

\section{October 2006}

This is a preprint of a paper intended for publication in a journal or proceedings. Since changes may be made before publication, this preprint should not be cited or reproduced without permission of the author. This document was prepared as an account of work sponsored by an agency of the United States Government. Neither the United States Government nor any agency thereof, or any of their employees, makes any warranty, expressed or implied, or assumes any legal liability or responsibility for any third party's use, or the results of such use, of any information, apparatus, product or process disclosed in this report, or represents that its use by such third party would not infringe privately owned rights. The views expressed in this paper are not necessarily those of the United States Government or the sponsoring agency. 


\section{QUANTITATIVE DETERMINATION OF LATERAL MODE DISPERSION IN FILM BULK ACOUSTIC RESONATORS THROUGH LASER ACOUSTIC IMAGING}

\author{
Ken L. Telschow \\ Idaho National Laboratory \\ Idaho Falls, ID 83415-2209 \\ kenneth.telschow@inl.gov
}

\author{
John D. Larson III \\ Avago Technologies, Inc. \\ San Jose, CA 95131 \\ john.larson@avagotech.com
}

\begin{abstract}
Film Bulk Acoustic Resonators are useful for many signal processing applications. Detailed knowledge of their properties is needed to optimize their design for specific applications. The finite size of these resonators precludes their use in single acoustic modes; rather, multiple wave modes, such as, lateral wave modes are always excited concurrently. In order to determine the contributions of these modes, we have been using a newly developed full-field laser acoustic imaging approach to directly measure their amplitude and phase throughout the resonator.
\end{abstract}

This paper describes new results comparing modeling including both elastic and piezoelectric effects in the active material with imaging measurement of all excited modes. Fourier transformation of the acoustic amplitude and phase displacement images provides a quantitative determination of excited mode amplitude and wavenumber at any frequency. Images combined at several frequencies form a direct visualization of lateral mode excitation and dispersion for the device under test allowing mode identification and comparison with predicted operational properties. Discussion and analysis are presented for modes near the first longitudinal thickness resonance $(\sim 900 \mathrm{MHz})$ in an AIN thin film resonator. Plate wave modeling, taking account of material crystalline orientation, elastic and piezoelectric properties and overlayer metallic films, is discussed in relation to direct image measurements.

\section{INTRODUCTION}

Microscopic film bulk acoustic resonators (FBARs) are utilized in the telecommunications industry for a variety of signal processing and routing tasks. Direct measurements of the acoustic motion of these resonators provide additional characterization of the device's electrical operation. A new method for full-field viewing these acoustic motions at $\mathrm{GHz}$ frequencies, without scanning, was used that performs optical full image detection of the acoustic displacement amplitude and phase motion in a microscopic geometry using photorefractive dynamic holography. The apparatus and operation of this approach have been previously described for measuring UHF acoustical motion at a single point and imaging [1,2]. In this paper we describe results and analysis from imaging measurements of the lateral acoustic mode motion in a nearly square FBAR around its series resonance and compare with model predictions.

The FBAR resonator was a nearly square $160 \mu \mathrm{m}$ micromachined thin plate of Aluminum Nitride (AIN) coated with thin Molybdenum (Mo) metal electrodes exhibiting a series resonance at $875 \mathrm{MHz}$. The thickness of the Mo layers was $0.78 \mu \mathrm{m}$ (oriented with the (111) plane normal to the thickness direction) and the AlN layer $2.24 \mu \mathrm{m}(0001)$, for a total plate thickness of $3.80 \mu \mathrm{m}$. The AlN plate was grown on a silicon wafer, etched and supported at the edges to provide stress-free boundary conditions. In operation, the FBAR vibrates primarily in the plate thickness mode at the series resonance frequency, but also exhibits many lateral mode motions at nearby frequencies that can affect device performance. This paper identifies the lateral plate wave motions observed in the finite sized resonator through images of the motion as well as through dispersion properties of the modes. Electrode layers, anisotropic crystalline orientation and piezoelectric effects are taken into account in the modeling calculation.

\section{IMAGING MEASUREMENTS}

Images of the resonator surface are combined interferometrically with a reference beam in a photorefractive crystal in a manner such that images of the surface displacement at synchronized acoustic phase intervals are obtained. Operation at any frequency is achieved by tuning an electrooptic modulator to that frequency offset by $1 / 4$ of the imaging capture frequency of the video camera. A sequence of 4 images phase shifted by $90^{\circ}$ is recorded at a rate of $5 \mathrm{~Hz}$ (video camera running at 20 $\mathrm{Hz}$ ). Images are subtracted from their $180^{\circ}$ counterparts to produce both the inphase and quadrature images of the acoustic motion, allowing complete determination of acoustic vertical displacement amplitude and phase at all points on the surface and in the immediate vicinity of the FBAR on the substrate. Complete data acquisition at one 

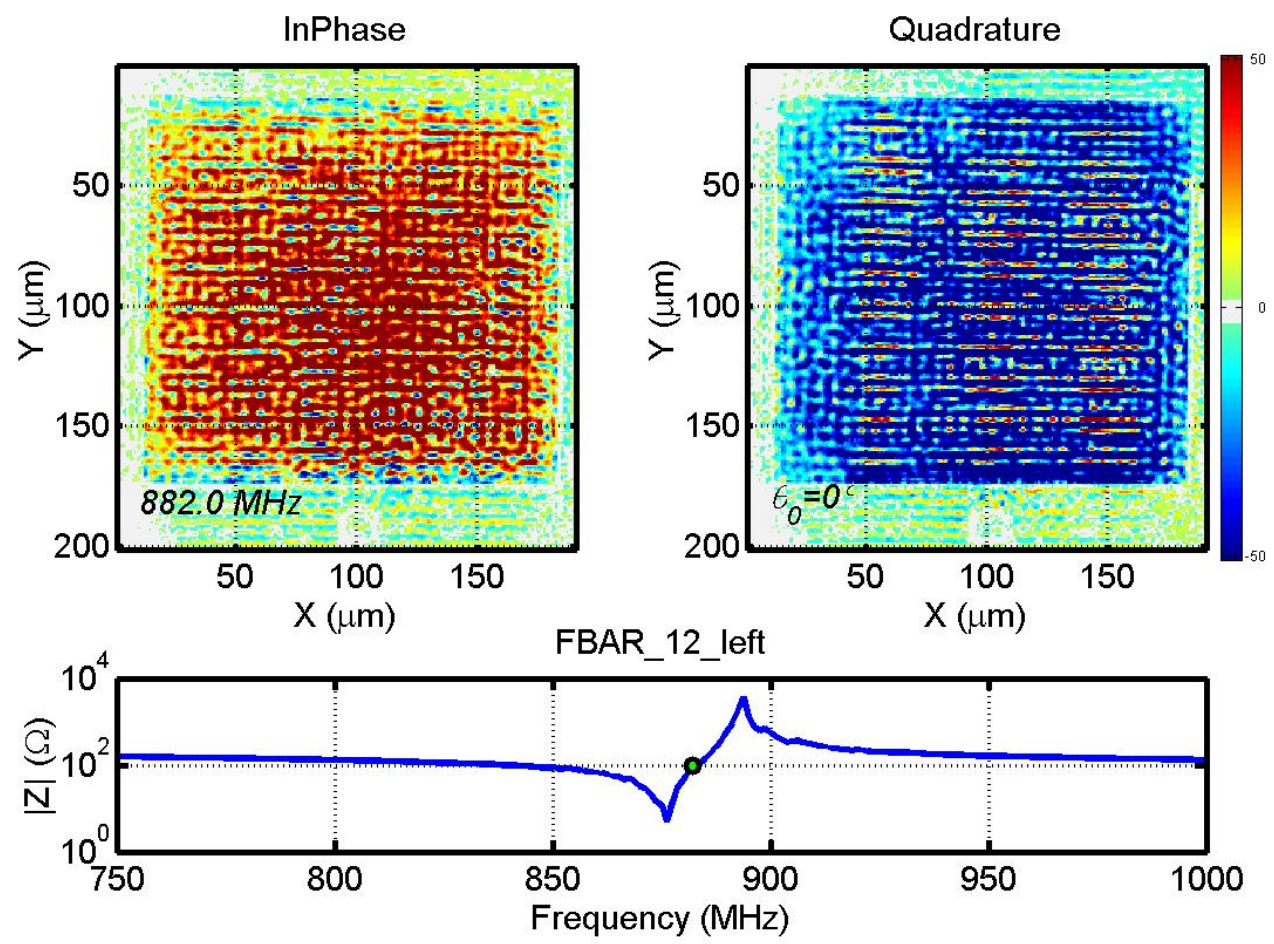

Figure 1. Measured inphase and quadrature acoustic displacement at $882 \mathrm{MHz}$ along with the electrical input impedance showing a primary resonance at $875 \mathrm{MHz}$. In the images, red represents positive displacement, blue negative and values near zero artificially made white so the sample outline is distinctly shown.

frequency of $256 \times 240$ pixel images (61440 spatial points) with 128 averages is achieved in less than 30 seconds providing rapid acoustical imaging.

Fig. 1 shows the two images obtained at a frequency well above the series resonance. The figure shows the inphase and quadrature acoustic normal displacement images that depict several patterns with different spatial wavelengths. The FBAR electrical impedance is also shown in the lower part of the figure along with the location of the measurement frequency. Both positive and negative displacements are represented in the figure with the scale shown at the right. Values near zero displacement are arbitrarily reproduced as white so that the sample outline is readily visible. From these images alone, one can pick out at least two spatial wavelengths.

\section{LATERAL MODE IMAGE ANALYSIS}

Fig. 2 shows Fast Fourier Transform (FT) analysis of the lateral mode images at frequencies below, at, and above the series resonance. The FT images show that there are modes with at least four different wavelengths in addition to the large series resonance motion at the center, all excited simultaneously. In these figures, the spatial dimension is determined by calibrating the displacement image with known lateral translations from an XY micrometer stage. A striking feature, seen in the FT images, is that the response of all modes exhibits circular symmetry with respect to the XY plane. This symmetry is a result of the FBAR fabrication process that is designed to produce AIN in the hexagonal (Wurtzite) phase with the c-axis perpendicular to the plate surface. Materials with hexagonal symmetry exhibit isotropic symmetry of all mode wave speeds in the XY plane [3].

It is evident from the figure that several modes are present that change wavelength $(\lambda)$ as the frequency increases. In particular, the figure at $765 \mathrm{MHz}$ exhibits 3 modes that generally increase in wavenumber $(v=1 / \lambda)$ with frequency. Three prominent modes are exhibited in the crosssection of the FT magnitude along the x-axis shown in Fig. 3. Since the material exhibits circular symmetry, the results displayed are averages over all angles in the XY plane. Continuing this process for all the frequencies measured in the range from $750 \mathrm{MHz}$ to $1000 \mathrm{MHz}$ provides a visual mapping of the mode dispersion [4]. The existence of these modes comes from the resonator being finite in size and the motion from all the modes is needed to satisfy the boundary conditions. Three very prominent modes are displayed in the figure and a fourth is sometimes visible for frequencies below $840 \mathrm{MHz}$ (see $765 \mathrm{MHz}$ image in Fig. 2). The identity of these modes becomes apparent when the dispersion curve is displayed as shown in Fig. 4 from a compilation of crosssections at several frequencies. This image represents an absolute measurement of mode dispersion relying only on the determination of image dimensions and frequency for 

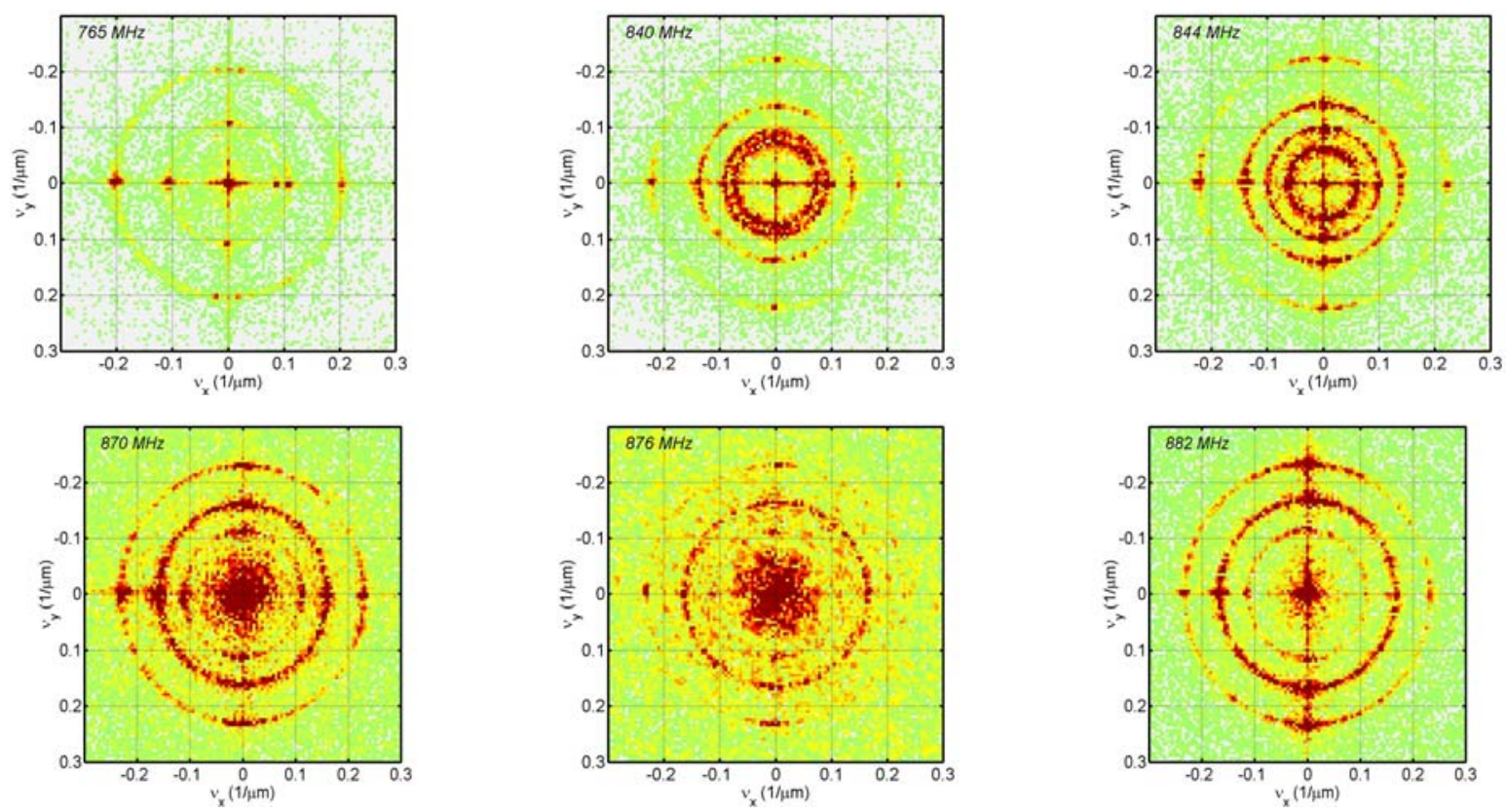

Figure 2. Acoustic displacement Fourier Transform magnitude images near the primary resonance

accuracy. The resulting image of the mode dispersion shown in Fig. 4 quick and straight forward method of identifying and mapping out lateral wave modes in the FBAR and other similar structures involving wave motion at the surface.

\section{Lateral Mode Dispersion Curve Calculation}

A calculation of the wave mode dispersion relation for the multilayered plate was performed using partial wave analysis. This method orients the material so that the given crystalline plane is normal to the plate surface, then the three elastic wave modes are found that have a phase velocity in the planar propagation direction, [001], equal to a given value for a given frequency. Linear combinations of these modes are formed that then match the boundary conditions of continuity of the displacement-traction vector at the layer interfaces. This approach is repeated for all three layers and

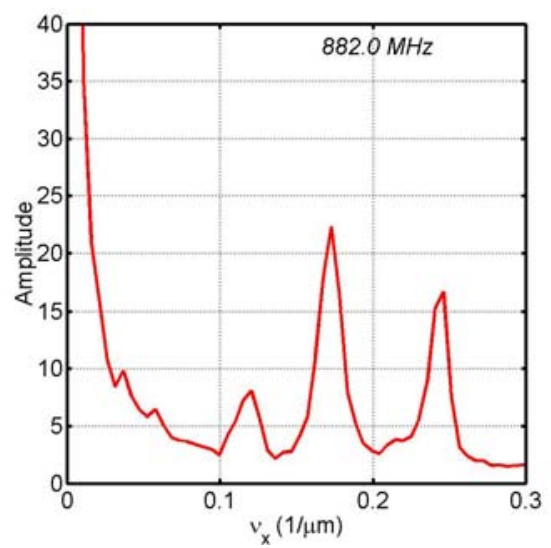

Figure 3. Fourier Transform average magnitude at $882 \mathrm{MHz}$ the stress-free boundary condition at the outer surfaces is inserted resulting in a set of linear equations that must be solved simultaneously. The dispersion relations are then the values of frequency and wavenumber that set the determinant of the global matrix equal to zero, as described in [5]. This method allows use of any material in any layer for which the density, elastic stiffness, piezoelectric stress and dielectric constants as well as crystalline orientation are known. Results of the modeling are shown in Fig. 5 by the dashed lines for the two lowest symmetric $\left(\mathrm{S}_{0}, \mathrm{~S}_{1}\right)$ and antisymmetric $\left(\mathrm{A}_{0}, \mathrm{~A}_{1}\right)$ modes compared to that measured from Fig. 4. The AlN layer is oriented with the c-axis perpendicular to the plate surface (0001) and the Mo layers are oriented in the (111) plane. The elastic coefficients, density, piezoelectric stress constants and dielectric permittivity used for the AlN structure were taken from [6] and that for Mo from [7]. The boundary conditions used for

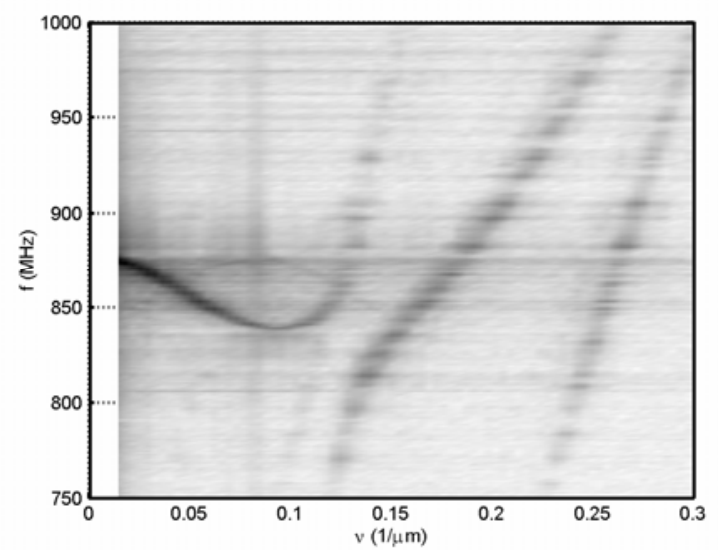

Figure 4. Image formed of the lateral wave mode dispersion from the cross-sections of the FT images over a range of frequencies. 


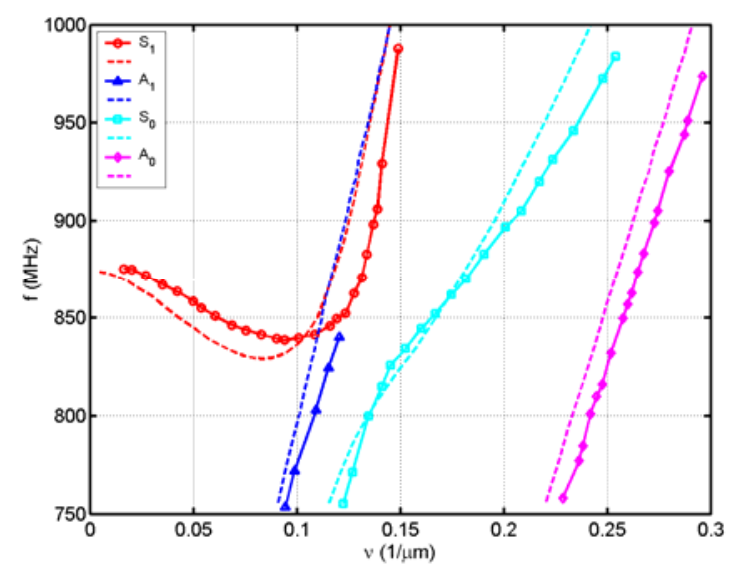

Figure 5. Comparison of the lateral wave mode dispersion from the FT images (points/solid lines) to the calculation of the first four Lamb modes in a Mo/AlN/Mo layered plate (dashed lines).

the calculation were that the normal stress and free charge were assumed zero at the outer layer plate surfaces.

The modeling predicts a total plate thickness of $3.98 \mu \mathrm{m}$ by normalizing to the series resonance frequency to 875 $\mathrm{MHz}$. This thickness value compares reasonably well to the experimental total plate thickness of $3.80 \mu \mathrm{m}$. The graph shows dispersion curves that allow identification of the modes as the lowest four Lamb wave modes, as predicted. The discrepancy between the measured and calculated curves is reasonable given that the plate is not infinite in lateral extent. Including finite plate lateral boundaries that also satisfy electrical and elastic boundary conditions can affect the dispersion curve [8] and is the topic of further study. The discrepancy can also be due to the actual material properties being slightly different than the published values used in the calculation. Optimization of the fit between measurement and calculation would yield a better estimate of the actual parameters for this FBAR; however, this might require a different approach from the analytical method utilized for this work.

\section{SUMMARY}

FBAR measurements have been presented using a fullfield imaging method that rapidly measures the acoustic amplitude and phase over the entire surface simultaneously. Detailed analysis of the images in the Fourier transform domain identifies the lateral modes observed as $\mathrm{S}_{0}, \mathrm{~S}_{1}$ and $\mathrm{A}_{0}, \mathrm{~A}_{1}$ Lamb waves. Reasonably good comparison between the measured and calculated dispersion curves was found with a multilayer model that accounts for crystalline orientation and includes piezoelectric coupling in the AIN layer as well as the orientation of the Mo electrode layers.

\section{ACKNOWLEDGMENT}

This work was sponsored by the U.S. Department of Energy, Basic Energy Sciences under DOE Idaho Operations
Office Contract

DE-AC07-05ID14517 and

Avago

Technologies Inc.

\section{REFERENCES}

[1] K. L. Telschow, V. A. Deason, D. L. Cottle, and J. D. Larson III, "Full-Field Imaging Of GHz Film Bulk Acoustic Resonator Motion," IEEE Trans. Ultrason., Ferroelect., Freq. Contr. 50 (10), 1279-1285 (2003).

[2] K. L. Telschow, V. A. Deason, R. S. Schley, and S. M. Watson, "Direct Imaging of Lamb Waves in Plates using Photorefractive Dynamic Holography," J. Acoust. Soc. Am. 106 (5), 2578-2587 (1999).

[3] B. A. Auld, Acoustic Fields and Waves in Solids (R.E. Krieger, Malabar, Fl., 1990) Vol. I, 396-398.

[4] T. Pensala, T. Makkonen, J. Vartiainen, J. Knuutila, J. Kaitila, O. Holmgren and M. M. Salomaa, "Laser Interferometric Measurement of Lamb Wave Dispersion and Extraction of Material Parameters in FBARs," 2002 IEEE Ultrasonics Symposium Proceedings, S. C. Schneider, D. E. Yuhas eds., IEEE, Piscataway, NJ. Vol.1, 977-980 (2002).

[5] O. M. Mukdadi, S. K. Datta, K. L. Telschow and V. A. Deason,"Ultrasonic Guided Waves in Thin Orthotropic Layers: Theoretical Analysis and Dynamic Holographic Imaging Measurement," IEEE Trans. Ultrason., Ferroelect., Freq. Contr. 48 (6) $1581-1593$ (2001).

[6] K. Tsubouchi \& N. Mikishiba, "Zero-temperature-coefficient SAW devices in AlN epitaxial films" "IEEE Trans. Sonics Ultr. SU-32(5), 634-644 (1985).

[7] J. M. Dickinson and P. E. Armstrong, "Temperature dependence of the elastic constants of Molybdenum," J. Appl. Phys. 38, 602-606 (1967)

[8] O. M. Mukdadi, Y. M. Desai, S. K. Datta, A. H. Shah and A. J. Niklasson, "Elastic guided waves in a layered plate with rectangular cross section,” J. Acoust. Soc. Am 112(5), 1766-1779 (2002). 